\title{
Adding insult to injury
}

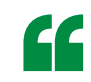

trypomastigotes

damage the host

cells plasma

membrane to

trigger cell entry

via endocytosis.
Eukaryotic cells can repair plasma membrane damage through a mechanism that relies on the influx of extracellular $\mathrm{Ca}^{2+}$; this triggers the fusion of lysosomes with the plasma membrane, resulting in the secretion of acid sphingomyelinase. In turn, this enzyme cleaves sphingomyelin in the outer leaflet of the membrane, generating ceramide, which creates increased inward curvature of the membrane, thereby supporting endocytosis of the damaged membrane. Writing in the Journal of Experimental Medicine, Fernandes et al. now show that Trypanosoma cruzi subverts this membrane repair pathway to invade the host cell.

Previous work has shown that the trypomastigote form of T. cruzi infects cells in a manner that depends on $\mathrm{Ca}^{2+}$-dependent fusion of the lysosome and the plasma membrane in the host cell. Such similarity to

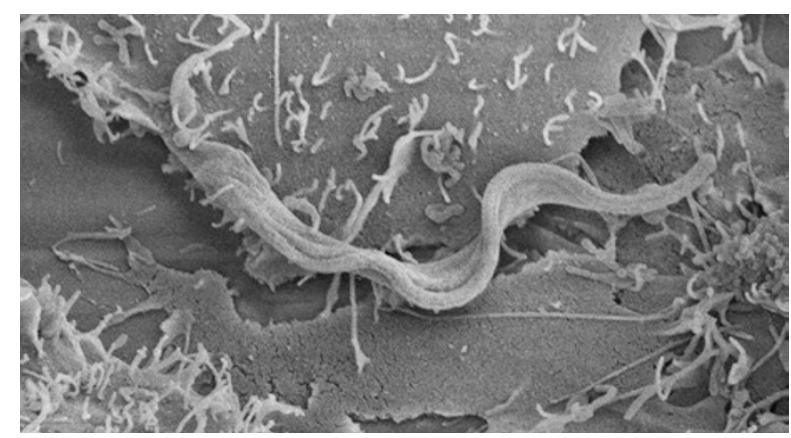

Scanning electron micrograph of a Trypanosoma cruzi trypomastigote attached to the surface of a cell through its posterior end. Image courtesy of R. Mortara, Federal University of São Paulo (UNIFESP), Brazil, and N. Andrews, University of Maryland, USA. events that occur during plasma membrane repair led Fernandes et al. to investigate whether this repair pathway played a part in parasite entry. They performed invasion assays in the presence or absence of $\mathrm{Ca}^{2+}$ (permissive and non-permissive conditions for repair, respectively) and observed that in the absence of $\mathrm{Ca}^{2+}$, the number of trypomastigotes invading HeLa cells decreased. To determine whether trypomastigotes were causing membrane damage during the invasion process, the authors added a membrane-impermeable dye, propidium iodide (PI), to the invasion assays. Although no PI uptake was observed during invasion in the presence of $\mathrm{Ca}^{2+}$, PI entered the cell in the absence of $\mathrm{Ca}^{2+}$, suggesting that trypomastigotes injure the plasma membrane but that the wounds are rapidly resealed in a $\mathrm{Ca}^{2+}$-dependent manner.

The authors next investigated the importance of lysosomal fusion and delivery of acid sphingomyelinase to the cell surface during invasion. They observed that trypomastigotes stimulated substantial translocation of a fluorescently tagged version of lysosomal-associated membrane protein 1 (LAMP1) to the surface of HeLa cells, and also stimulated endocytosis. Furthermore, trypomastigote infection was inhibited by blocking acid sphingomyelinase activity with desipramine or silencing acid sphingomyelinase expression with RNA interference. Taken together, these data suggest that trypomastigotes damage the plasma membrane of host cells to trigger cell entry via endocytosis.

So how does T. cruzi damage the plasma membrane in the first place? Trypomastigotes secrete a poreforming toxin, TcTox, to escape from the parasitophorous vacuole into the cytoplasm. However, this toxin only permeabilizes membranes at the acidic $\mathrm{pH}$ found in the parasitophorous vacuole, making it unlikely to have a role in plasma membrane damage. Instead, the authors propose that mechanical forces imposed by the attachment of parasites to the cell surface might damage the membrane. Consistent with this idea, the authors observed that trypomastigotes strongly attach to host cells through their posterior end, meaning that flagellar motility would propel the parasites away from the cell, perhaps damaging the membrane in the process.

Andrew Jermy

ORIGINAL RESEARCH PAPER Fernandes,

M. C. et al. Trypanosoma cruzi subverts the sphingomyelinase-mediated plasma membrane repair pathway for cell invasion.J. Exp. Med. 208, 909-921 (2011)

FURTHER READING Andrade, L. O. \& Andrews, N. W. The Trypanosoma cruzi-host-cell interplay: location, invasion, retention. Nature Rev. Microbiol. 3, 819-823 (2005) 